\title{
ANALYSIS OF THE QUEUING NETWORK WITH MESSAGES BYPASS OF SYSTEMS IN TRANSIENT BEHAVIOR
}

\author{
Mikhail Matalytski ${ }^{1}$, Victor Naumenko ${ }^{2}$ \\ ${ }^{I}$ Institute of Mathematics, Czestochowa University of Technology, Poland \\ ${ }^{2}$ Faculty of Mathematics and Computer Science, Grodno State University, Belarus \\ m.matalytski@gmail.com,victornn86@gmail.com
}

\begin{abstract}
The article provides a survey of an open exponential network with a multiline queuing systems (QS) with a bypass service messages in the transient behavior. Messages with some probability join the QS, and with an additional probability to move immediately to another QS or leave the network. The paper describes the methodology finding the timedependent state probabilities of the network of its kind in the transient state. To find the state probabilities of the network, the method multivariate generating functions was applied. Examples are considered.
\end{abstract}

\section{Introduction}

Currently, development of information and telecommunication networks (TCN) is significant. The issue of fair and full satisfaction of the requirements of their users is wide. In practice, often there are situations when user makes the message to the TCN site, assesses how much time they will have to wait or how many user messages in front of them in line, and, according to this estimate is expected, or sends a request to another node of TCN. Such a situation, for example, may occur in service centres or points of collective use (PCU). A customer arriving at one of PCU, assesses how much time will be needed to wait in line and decides to move to the next free operator to service your message, or to the operator with the smallest queue of customers. The client can also leave this PCU to another.

The probabilistic models of such objects could be queuing networks (QN) with Bypass Systems Service (QS) messages. To reduce the workload of the QS and more uniform distribution of load on the network can be used in different ways, one of which is - the introduction of probabilistic rounds QS messages. This model, in particular, to consider the limits on the number of bids or the estimated waiting time in queuing messages, as well as restrictions on the acceptance of messages coming from the others defined QS. 


\section{Formulation of the problem}

Consider an open exponential QN of an arbitrary structure consisting of $n$ QS, enumerated by numbers from 1 to $n$. Messages have a chance to join the queue, and with an additional probability to move immediately in accordance with the transition probability matrix of the other QS, or leave the network. The probability of joining the QS depends on the state of the QS and the number of QS with which the messages are sent to this QS. It is assumed that the incoming flow of applications to the network is simple. The results of studies of such networks in the steady state are given in [1-4]. This paper describes a method of finding the timedependent state probabilities of the network of such a network in the transient state.

Let $m_{i}$ - the number of identical service lines in the QS $S_{i}, I_{i}$ - a vector of dimension $n$, consisting of zeros except the $i$-th component, which is equal to 1 , $i=\overline{1, n} ; p_{i j}$ - the transition probability of the message after service in the system $S_{i}$ into the system $S_{j}, i, j=\overline{0, n}$, we assume the system $S_{0}$ is the external environment. Let us consider the case when the parameters of the incoming flow of messages and services depend on time, i.e. the time interval $[t, t+\Delta t)$ in the network receives an message with a probability $\lambda(t) \Delta t+o(\Delta t)$, and if at the time $t$ of service on the line $i$-th QS located in a message, at the range $[t, t+\Delta t)$ of its services will end with a probability $\mu_{i}(t) \Delta t+o(\Delta t), i=\overline{1, n}$. The message is sent to the $i$-th QS with probability $p_{0 i}, \sum_{i=1}^{n} p_{0 i}=1$. The message sent to this QS from the external environment at moment time $t$, with a probability $f^{(i)}(k, t)$, when the network is in a state $(k, t)$, joins the queue, and the probability $1-f^{(i)}(k, t)$ is not attached to the queue, regardless of the handled (i.e., it's time of service with a probability of 1 is equal to zero). If the message has been served in the $i$-th QS, it is likely to be sent immediately to the $j$-th QS with probability $p_{i j}$, and leaves the

QN with the probability $p_{i 0}, \sum_{j=0}^{n} p_{i j}=1, i=1, \ldots, n$.

Let $k(t)=(k, t)=\left(k_{1}, k_{2}, \ldots, k_{n}, t\right)$ - the state vector of the network, where $k_{i}$ - the number of messages at the moment $t$ in the system $S_{i}, i=\overline{1, n} ; \varphi_{i}(k, t)$ - the conditional probability that the message is delivered to the $i$-th QS at time $t$, when the network is in a state $(k, t)$, will not be serviced by any of the QS; $\psi_{i j}(k, t)$ - the conditional probability that the message is delivered to the $i$-th QS outside at time $t$, when the network is in state $(k, t)$, first time, a service in $j$-th QS; $\alpha_{i}(k, t)$ - the conditional probability that the message, served in the $i$-th queuing system at time 
$t$, when the network is in a state $(k, t)$, will no longer be served in any of QS; $\beta_{i j}(k, t)$ - the conditional probability that the message, served in the $i$-th queuing system at time $t$, when the network is in state $(k, t)$ for the first time then receive services in the $j$-th QS, $i, j=\overline{1, n}$.

According to the formula of total probability, we obtain:

$$
\begin{gathered}
\varphi_{i}(k, t)=\left(1-f^{(i)}(k, t)\right)\left(p_{i 0}+\sum_{j=1}^{n} p_{i j} \varphi_{j}(k, t)\right), i=\overline{1, n}, \\
\psi_{i j}(k, t)=f^{(i)}(k, t) \delta_{i j}+\left(1-f^{(i)}(k, t)\right) \sum_{l=1}^{n} p_{i l} \psi_{l j}(k, t), i, j=\overline{1, n}, \\
\alpha_{i}(k, t)=p_{i 0}+\sum_{j=1}^{n} p_{i j} \varphi_{j}\left(k-I_{i}, t\right), i=\overline{1, n}, \\
\beta_{i j}(k, t)=\sum_{l=1}^{n} p_{i l} \psi_{l j}\left(k-I_{i}, t\right), i, j=\overline{1, n},
\end{gathered}
$$

where $\delta_{i j}$ - the Kronecker delta. We have the equalities

$$
\alpha_{i}(k, t)=1-\sum_{j=1}^{n} \beta_{i j}(k, t), \varphi_{i}(k, t)+\sum_{j=1}^{n} \psi_{i j}(k, t)=1, i, j=\overline{1, n} .
$$

From (1) and (3) we find

$$
\psi_{i j}(k, t)=f^{(i)}(k, t) \delta_{i j}+\left(1-f^{(i)}(k, t)\right) \beta_{i j}\left(k-I_{i}, t\right), i, j=\overline{1, n} .
$$

Lemma 1. The probabilities of states of the considered network satisfy the difference-differential equations (DDE):

$$
\begin{gathered}
\frac{d P(k, t)}{d t}=-\sum_{i=1}^{n}\left[\lambda(t) p_{0 i}\left(1-\varphi_{i}(k, t)\right)+\mu_{i}(t)\left(1-\beta_{i i}(k, t)\right) \min \left(m_{i}, k_{i}\right)\right] P(k, t)+ \\
+\lambda(t) \sum_{i=1}^{n} \sum_{j=1}^{n} p_{0 i} \psi_{i j}\left(k-I_{j}, t\right) u\left(k_{i}\right) P\left(k-I_{j}, t\right)+ \\
\quad+\sum_{i=1}^{n} \mu_{i}(t) \min \left(m_{i}, k_{i}+1\right) \alpha_{i}\left(k+I_{i}, t\right) P\left(k+I_{i}, t\right)+ \\
+\sum_{\substack{i, j=1 \\
i \neq j}}^{n} \mu_{i}(t) \min \left(m_{i}, k_{i}+1\right) \beta_{i j}\left(k+I_{i}-I_{j}, t\right) u\left(k_{j}\right) P\left(k+I_{i}-I_{j}, t\right),
\end{gathered}
$$

where $u(x)=\left\{\begin{array}{l}1, x>0 \\ 0, x \leq 0\end{array}\right.$ - the Heaviside function. 
Proof. In view of the exponential service times of applications a random process $k(t)=(k, t)$ is a Markov chain with a countable number of states. The possible transitions in the state $(k, t+\Delta t)$ for the time $\Delta t$ :

a) from the state $\left(k-I_{j}, t\right)$ with the probability

$$
\lambda(t) p_{0 i} \psi_{i j}\left(k-I_{j}, t\right) u\left(k_{i}\right) \Delta t+o(\Delta t), i=\overline{1, n} ;
$$

b) from the state $\left(k+I_{i}, t\right)$ with the probability

$$
\mu_{i}(t) \min \left(m_{i}, k_{i}+1\right) \alpha_{i}\left(k+I_{i}, t\right) \Delta t+o(\Delta t), i=\overline{1, n} ;
$$

c) from the state $\left(k+I_{i}-I_{j}, t\right)$ with the probability

$$
\mu_{i}(t) \min \left(m_{i}, k_{i}+1\right) \beta_{i j}\left(k+I_{i}-I_{j}, t\right) u\left(k_{j}\right) \Delta t+o(\Delta t), i, j=\overline{1, n} ;
$$

d) from the state $(k, t)$ with the probability

$$
1-\left[\lambda(t) p_{0 i}\left(1-\phi_{i}(k, t)\right)+\mu_{i}(t)\left(1-\beta_{i i}(k, t)\right) \min \left(m_{i}, k_{i}\right)\right] \Delta t+o(\Delta t) ;
$$

e) of the remaining states with a probability $o(\Delta t)$.

Then, using the formula of total probability, we can obtain

$$
\begin{gathered}
P(k, t+\Delta t)=\lambda(t) \sum_{i=1}^{n} \sum_{j=1}^{n} p_{0 i} \psi_{i j}\left(k-I_{j}, t\right) u\left(k_{i}\right) P\left(k-I_{j}, t\right) \Delta t+ \\
+\sum_{i=1}^{n} \mu_{i}(t) \min \left(m_{i}, k_{i}+1\right) \alpha_{i}\left(k+I_{i}, t\right) P\left(k+I_{i}, t\right) \Delta t+ \\
+\sum_{i, j=1}^{n} \mu_{i}(t) \min \left(m_{i}, k_{i}+1\right) \beta_{i j}\left(k+I_{i}-I_{j}, t\right) u\left(k_{j}\right) P\left(k+I_{i}-I_{j}, t\right) \Delta t+ \\
+P(k, t)\left\{1-\sum_{i=1}^{n}\left[\lambda(t) p_{0 i}\left(1-\varphi_{i}(k, t)\right)+\mu_{i}(t)\left(1-\beta_{i i}(k, t)\right) \min \left(m_{i}, k_{i}\right)\right] \Delta t\right\}+o(\Delta t) .
\end{gathered}
$$

Dividing both sides of this relationship by $\Delta t$ and taking the limit $\Delta t \rightarrow 0$, we obtain a system of equations for the state probabilities of the network (5).

\section{Finding the state probabilities using the method of multivariate generating functions}

Let $m_{i}=1, i=\overline{1, n}$, and suppose that all network systems operating in high load mode, i.e. $k_{i}(t)>0, \forall t>0, i=\overline{1, n}$, then the system (5) will take the form 


$$
\begin{aligned}
\frac{d P(k, t)}{d t}= & -\sum_{i=1}^{n}\left[\lambda(t) p_{0 i}\left(1-\varphi_{i}(k, t)\right)+\mu_{i}(t)\left(1-\beta_{i i}(k, t)\right)\right] P(k, t)+ \\
& +\lambda(t) \sum_{i=1}^{n} \sum_{j=1}^{n} p_{0 i} \psi_{i j}\left(k-I_{j}, t\right) P\left(k-I_{j}, t\right)+ \\
& +\sum_{i=1}^{n} \mu_{i}(t) \alpha_{i}\left(k+I_{i}, t\right) P\left(k+I_{i}, t\right)+ \\
+ & \sum_{\substack{i, j=1 \\
i \neq j}}^{n} \mu_{i}(t) \beta_{i j}\left(k+I_{i}-I_{j}, t\right) P\left(k+I_{i}-I_{j}, t\right) .
\end{aligned}
$$

Note that the number of equations in (6) is countable, when the network is open, and the final when it is closed.

We denote $\Psi_{n}(z, t), z=\left(z_{1}, z_{2}, \ldots, z_{n}\right), n$-dimensional generating function:

$$
\begin{aligned}
\Psi_{n}(z, t) & =\sum_{k_{1}=0}^{\infty} \sum_{k_{2}=0}^{\infty} \ldots \sum_{k_{n}=0}^{\infty} P\left(k_{1}, k_{2}, . ., k_{n}, t\right) z_{1}^{k_{1}} z_{2}^{k_{2}} \cdot \ldots \cdot z_{n}^{k_{n}}= \\
& =\sum_{k_{1}=1}^{\infty} \sum_{k_{2}=1}^{\infty} \ldots \sum_{k_{n}=1}^{\infty} P(k, t) \prod_{i=1}^{n} z_{i}^{k_{i}}
\end{aligned}
$$

the summation is taken over each $k_{i}, i=\overline{1, n}$, because the network operates in a high load mode.

Consider the case when the conditional probabilities $\varphi_{i}(k, t), \psi_{i j}(k, t), \alpha_{i}(k, t)$, $\beta_{i j}(k, t)$ do not depend on the state of the network.

Lemma 2. If at the initial moment of time $Q N$ is in a state $\left(x_{1}, x_{2}, \ldots, x_{n}, 0\right), x_{i}>0$, $i=\overline{1, n}$, then the expression for the generating function (7) can be rewritten as

$$
\begin{gathered}
\Psi_{n}(z, t)=C_{n}(z) \exp \left\{-\int \sum_{i=1}^{n}\left[\lambda(t) p_{0 i}\left(1-\varphi_{i}(t)\right)+\mu_{i}(t)\left(1-\beta_{i i}(t)\right)+\right.\right. \\
\left.\left.+\lambda(t) p_{0 i} z_{i} \sum_{\mathrm{j}=1}^{n} \psi_{i j}(t)+\mu_{i}(t) \frac{\alpha_{i}(t)}{z_{i}}+\mu_{i}(t) \sum_{j=1}^{n} \beta_{i j}(t) \frac{z_{j}}{z_{i}}\right] d t\right\} .
\end{gathered}
$$

the function $C_{n}(z)$ is defined in the proof and has the form (12).

Proof. Multiplying (6) by $\prod_{l=1}^{n} z_{l}^{k_{l}}$ and summing over all possible values of $k_{l}$ from $l$ to $+\infty, l=\overline{1, n}$, we get: 


$$
\begin{gathered}
\sum_{k_{1}=1}^{\infty} \ldots \sum_{k_{n}=1}^{\infty} \frac{d P(k, t)}{d t} \prod_{l=1}^{n} z_{l}^{k_{l}}= \\
=-\sum_{i=1}^{n}\left[\lambda(t) p_{0 i}\left(1-\varphi_{i}(t)\right)+\mu_{i}(t)\left(1-\beta_{i i}(t)\right)\right] \sum_{k_{1}=1}^{\infty} \ldots \sum_{k_{n}=1}^{\infty} P(k, t) \prod_{l=1}^{n} z_{l}^{k_{l}}+ \\
+\lambda(t) \sum_{i=1}^{n} \sum_{j=1}^{n} p_{0 i} \psi_{i j}(t) \sum_{k_{1}=1}^{\infty} \ldots \sum_{k_{n}=1}^{\infty} P\left(k-I_{j}, t\right) \prod_{l=1}^{n} z_{l}^{k_{l}}+\sum_{i=1}^{n} \mu_{i}(t) \alpha_{i}(t) \sum_{k_{1}=1}^{\infty} \ldots \sum_{k_{n}=1}^{\infty} P\left(k+I_{i}, t\right) \prod_{l=1}^{n} z_{l}^{k_{l}}+ \\
+\sum_{\substack{i, j=1 \\
i \neq j}}^{n} \mu_{i}(t) \beta_{i j}(t) \sum_{k_{1}=1}^{\infty} \ldots \sum_{k_{n}=1}^{\infty} P\left(k+I_{i}-I_{j}, t\right) \prod_{l=1}^{n} z_{l}^{k_{l}}
\end{gathered}
$$

Consider some of sums included in right side of (9). Let

$$
\sum_{1}(z, t)=\sum_{i=1}^{n} \sum_{j=1}^{n} p_{0 i} \psi_{i j}(t) \sum_{k_{1}=1}^{\infty} \ldots \sum_{k_{n}=1}^{\infty} P\left(k-I_{j}, t\right) \prod_{l=1}^{n} z_{l}^{k_{l}} .
$$

Then

$$
\begin{aligned}
& \sum_{1}(z, t)=\sum_{i=1}^{n} p_{0 i} z_{i} \sum_{j=1}^{n} \psi_{i j}(t) \sum_{\substack{k_{j}=1 \\
j=1, n, j \neq i}}^{\infty} \sum_{k_{i}=1}^{\infty} P\left(k-I_{j}, t\right) \prod_{\substack{l=1 \\
l \neq i}}^{n} z_{l}^{k_{l}} z_{i}^{k_{i}-1}= \\
= & \sum_{i=1}^{n} p_{0 i} z_{i} \sum_{\substack{\mathrm{j}=1 \\
j \neq i}}^{n} \psi_{i j}(t) \sum_{\substack{k_{j}=0 \\
j=1, n}}^{\infty} P(k, t) \prod_{l=1}^{n} z_{l}^{k_{l}}=\sum_{i=1}^{n} p_{0 i} z_{i} \sum_{\substack{\mathrm{j}=1 \\
j \neq i}}^{n} \psi_{i j}(t) \Psi_{n}(z, t) .
\end{aligned}
$$

For the sum $\sum_{2}(z, t)=\sum_{i=1}^{n} \mu_{i}(t) \alpha_{i}(t) \sum_{k_{1}=1}^{\infty} \ldots \sum_{k_{n}=1}^{\infty} P\left(k+I_{i}, t\right) \prod_{l=1}^{n} z_{l}^{k_{l}}$ we have:

$$
\begin{gathered}
\sum_{2}(z, t)=\sum_{i=1}^{n} \mu_{i}(t) \frac{\alpha_{i}(t)}{z_{i}} \sum_{k_{1}=1}^{\infty} \ldots \sum_{k_{n}=1}^{\infty} P\left(k+I_{i}, t\right) \prod_{\substack{l=1 \\
l \neq i}}^{n} z_{l}^{k_{l}} z_{i}^{k_{i}+1}= \\
=\sum_{i=1}^{n} \mu_{i}(t) \frac{\alpha_{i}(t)}{z_{i}} \sum_{\substack{k_{j}=0 \\
j=1, n, j \neq i}}^{\infty} \sum_{\substack{k_{i}=1 \\
\infty}}^{\infty} P(k, t) \prod_{\substack{l=1 \\
l \neq i}}^{n} z_{l}^{k_{l}} z_{i}^{k_{i}}= \\
=\sum_{i=1}^{n} \mu_{i}(t) \frac{\alpha_{i}(t)}{z_{i}} \Psi_{n}(z, t)-\sum_{i=1}^{n} \mu_{i}(t) \frac{\alpha_{i}(t)}{z_{i}} \sum_{\substack{k_{j}=1 \\
j=1, n, j \neq i}}^{\infty} P\left(k_{1}, \ldots, k_{i-1}, 0, k_{i+1} \ldots, k_{n}, t\right) \prod_{\substack{l=1 \\
l \neq i}}^{n} z_{l}^{k_{l}}= \\
=\sum_{i=1}^{n} \mu_{i}(t) \frac{\alpha_{i}(t)}{z_{i}} \Psi_{n}(z, t),
\end{gathered}
$$

because QS $S_{i}$ operates in a high load, i.e. $P\left(k_{1}, \ldots, k_{i-1}, 0, k_{i+1} . ., k_{n}, t\right)=0, i=\overline{1, n}$. 
And finally, for the last sum

$$
\sum_{3}(z, t)=\sum_{\substack{i, j=1 \\ i \neq j}}^{n} \mu_{i}(t) \beta_{i j}(t) \sum_{k_{1}=1}^{\infty} \ldots \sum_{k_{n}=1}^{\infty} P\left(k+I_{i}-I_{j}, t\right) \prod_{l=1}^{n} z_{l}^{k_{l}}
$$

we will have:

$$
\begin{gathered}
\sum_{3}(z, t)=\sum_{\substack{i, j=1 \\
i \neq j}}^{n} \mu_{i}(t) \beta_{i j}(t) \frac{z_{j}}{z_{i}} \times \\
\times \sum_{\substack{k_{m}=1 \\
m=1, n, m \neq j}}^{\infty} \sum_{k_{j}=1}^{\infty} P\left(k_{1}, \ldots, k_{i-1}, k_{i}+1, k_{i+1}, \ldots, k_{j-1}, k_{j}-1, k_{j+1}, \ldots, k_{n}, t\right) \times \\
\times \prod_{\substack{l=1 \\
l \neq i, j}}^{n} z_{l}^{k_{l}} z_{i}^{k_{i}+1} z_{j}^{k_{j}-1}=\sum_{i, j=1}^{n} \mu_{i}(t) \beta_{i j}(t) \frac{z_{j}}{z_{i}} \sum_{\substack{k_{m}=1 \\
m=1, n, m \neq i}}^{\infty} \sum_{k_{i}=1}^{\infty} P(k, t) \prod_{l=1}^{n} z_{l}^{k_{l}}= \\
=\sum_{\substack{i, j=1 \\
i \neq j}}^{n} \mu_{i}(t) \beta_{i j}(t) \frac{z_{j}}{z_{i}} \Psi_{n}(z, t)-\sum_{\substack{i, j=1 \\
i \neq j}}^{n} \mu_{i}(t) \beta_{i j}(t) \frac{z_{j}}{z_{i}} \sum_{\substack{k_{m}=1 \\
m=1, n, m \neq j}}^{\infty} P\left(k_{1}, \ldots, k_{i-1}, 0, k_{i+1}, \ldots, k_{n}, t\right) \prod_{\substack{l=1 \\
l \neq i}}^{n} z_{l}^{k_{l}}= \\
=\sum_{\substack{i, j=1 \\
i \neq j}}^{n} \mu_{i}(t) \beta_{i j}(t) \frac{z_{j}}{z_{i}} \Psi_{n}(z, t),
\end{gathered}
$$

for the same reasons as for the sum $\sum_{2}(z, t)$.

Thus, to obtain the generating function of the homogeneous linear control

$$
\begin{gathered}
\frac{d \Psi_{n}(z, t)}{d t}=-\sum_{i=1}^{n}\left[\lambda(t) p_{0 i}\left(1-\varphi_{i}(t)\right)+\mu_{i}(t)\left(1-\beta_{i i}(t)\right)+\right. \\
\left.+\lambda(t) p_{0 i} z_{i} \sum_{j=1}^{n} \psi_{i j}(t)+\mu_{i}(t) \frac{\alpha_{i}(t)}{z_{i}}+\mu_{i}(t) \sum_{j=1}^{n} \beta_{i j}(t) \frac{z_{j}}{z_{i}}\right] \Psi_{n}(z, t) .
\end{gathered}
$$

Its solution has the form

$$
\begin{gathered}
\Psi_{n}(z, t)=C_{n}(z) \exp \left\{-\int \sum_{i=1}^{n}\left[\lambda(t) p_{0 i}\left(1-\varphi_{i}(t)\right)+\mu_{i}(t)\left(1-\beta_{i i}(t)\right)+\right.\right. \\
\left.\left.+\lambda(t) p_{0 i} z_{i} \sum_{j=1}^{n} \psi_{i j}(t)+\mu_{i}(t) \frac{\alpha_{i}(t)}{z_{i}}+\mu_{i}(t) \sum_{j=1}^{n} \beta_{i j}(t) \frac{z_{j}}{z_{i}}\right] d t\right\} .
\end{gathered}
$$


We shall assume that at the initial moment network is in a state

$$
\begin{gathered}
\left(x_{1}, x_{2}, \ldots, x_{n}, 0\right), x_{i}>0, i=\overline{1, n}, P\left(x_{1}, x_{2}, \ldots, x_{n}, 0\right)=1, \\
P\left(k_{1}, k_{2}, \ldots, k_{n}, 0\right)=0, \forall x_{i} \neq k_{i}, i=\overline{1, n} .
\end{gathered}
$$

Then the initial condition for this equation (11) will be

$$
\Psi_{n}(z, 0)=P\left(x_{1}, x_{2}, \ldots, x_{n}, 0\right) \prod_{l=1}^{n} z_{l}^{x_{l}}=\prod_{l=1}^{n} z_{l}^{x_{l}} .
$$

Using this initial condition, we obtain

$$
\begin{aligned}
C_{n}(z)=\exp \left\{\int\right. & {\left[\sum_{i=1}^{n}\left(\lambda(t) p_{0 i}\left(1-\varphi_{i}(t)\right)+\mu_{i}(t)\left(1-\beta_{i i}(t)\right)\right)+\lambda(t) \sum_{i=1}^{n} p_{0 i} z_{i} \sum_{j=1}^{n} \psi_{i j}(t)+\right.} \\
& \left.\left.+\sum_{i=1}^{n} \mu_{i}(t) \frac{\alpha_{i}(t)}{z_{i}}+\sum_{i, j=1}^{n} \mu_{i}(t) \beta_{i j}(t) \frac{z_{j}}{z_{i}}\right]\left.d t\right|_{t=0}\right\} \prod_{i=1}^{n} z_{l}^{x_{l}}
\end{aligned}
$$

Lemma is proved.

Then consider the case when probabilities $\varphi_{i}(t), \psi_{i j}(t), \alpha_{i}(t), \beta_{i j}(t)$ does not depend on time $i, j=\overline{1, n}$, in this case from (8) and (12) follows that

$$
\begin{aligned}
\Psi_{n}(z, t)= & \exp \left\{-\left[(\Lambda(t)-\Lambda(0)) \sum_{i=1}^{n} p_{0 i}\left(1-\varphi_{i}\right)+\sum_{i=1}^{n}\left(M_{i}(t)-M_{i}(0)\right)\left(1-\beta_{i i}\right)+\right.\right. \\
+(\Lambda(t)- & \Lambda(0)) \sum_{i=1}^{n} p_{0 i} z_{i} \sum_{\mathrm{j}=1}^{n} \psi_{i j}+\sum_{i=1}^{n}\left(M_{i}(t)-M_{i}(0)\right) \frac{\alpha_{i}}{z_{i}}+ \\
& \left.\left.+\sum_{i, j=1}^{n}\left(M_{i}(t)-M_{i}(0)\right) \beta_{i j} \frac{z_{j}}{z_{i}}\right]\right\} \prod_{i=1}^{n} z_{l}^{x_{l}}
\end{aligned}
$$

where $\Lambda(t)=\int \lambda(t) d t, M_{i}(t)=\int \mu_{i}(t) d t, i=\overline{1, n}$.

This expression can be rewritten as

$$
\begin{gathered}
\Psi_{n}(z, t)=a_{0}(t) \exp \left\{(\Lambda(t)-\Lambda(0)) \sum_{i=1}^{n} p_{0 i} z_{i} \sum_{j=1}^{n} \psi_{i j}\right\} \exp \left\{\sum_{i=1}^{n}\left(M_{i}(t)-M_{i}(0)\right) \frac{\alpha_{i}}{z_{i}}\right\} \times \\
\times \exp \left\{\sum_{i, j=1}^{n}\left(M_{i}(t)-M_{i}(0)\right) \beta_{i j} \frac{z_{j}}{z_{i}}\right\} \prod_{l=1}^{n} z_{l}^{x_{l}} \\
a_{0}(t)=\exp \left\{-\left((\Lambda(t)-\Lambda(0)) \sum_{i=1}^{n} p_{0 i}\left(1-\varphi_{i}\right)+\sum_{i=1}^{n}\left(M_{i}(t)-M_{i}(0)\right)\left(1-\beta_{i i}\right)\right)\right\}
\end{gathered}
$$


Transform (13) to a form suitable for finding the state probabilities of the network, expanding its exponential member in a Maclaurin series. Then the following statement is:

Theorem. The expression for the generating function (7) has the form

$$
\begin{gathered}
\Psi_{n}(z, t)=a_{0}(t) \sum_{l_{1}=0}^{\infty} \ldots \sum_{l_{n}=0}^{\infty} \sum_{q_{1}=0}^{\infty} \ldots \sum_{q_{n}=0}^{\infty} \sum_{\eta_{1}=0}^{\infty} \ldots \sum_{r_{n}=0}^{\infty}(\Lambda(t)-\Lambda(0))^{\sum_{i=1}^{n} l_{i}} \times \\
\times \prod_{i=1}^{n}\left[\frac{p_{0 i}^{l_{i}}\left(\prod_{j=1}^{n} \psi_{i j}\right)^{l_{i}} \alpha_{i}^{q_{i}}\left(\prod_{j=1}^{n} \beta_{i j}\right)^{r_{i}}}{l_{i} ! q_{i} ! r_{i} !}\left(M_{i}(t)-M_{i}(0)\right)^{q_{i}+r_{i}} z_{i}^{x_{i}+l_{i}-q_{i}-r_{i}+R}\right],
\end{gathered}
$$

where $R=\sum_{i=1}^{n} r_{i}$.

Proof. From (13) and Lemma 2 it follows that

$$
\Psi_{n}(z, t)=a_{0}(t) a_{1}(z, t) a_{2}(z, t) a_{3}(z, t) \prod_{l=1}^{n} z_{l}^{x_{l}},
$$

where

$$
\begin{gathered}
a_{1}(z, t)=\exp \left\{(\Lambda(t)-\Lambda(0)) \sum_{i=1}^{n} p_{0 i} z_{i} \sum_{j=1}^{n} \psi_{i j}\right\}=\prod_{i=1}^{n} \prod_{j=1}^{n} \exp \left\{(\Lambda(t)-\Lambda(0)) z_{i} p_{0 i} \psi_{i j}\right\}= \\
=\prod_{i=1}^{n} \prod_{j=1}^{n} \sum_{l_{i}=0}^{\infty} \frac{\left[(\Lambda(t)-\Lambda(0)) z_{i} p_{0 i} \psi_{i j}\right]^{t_{i}}}{l_{i} !}=\sum_{l_{1}=0}^{\infty} \ldots \sum_{l_{n}=0}^{\infty} \prod_{i=1}^{n} \prod_{j=1}^{n} \frac{\left[(\Lambda(t)-\Lambda(0)) z_{i} p_{0 i} \psi_{i j}\right]^{j_{i}}}{l_{i} !}= \\
=\sum_{l_{1}=0}^{\infty} \ldots \sum_{l_{n}=0}^{\infty} \frac{(\Lambda(t)-\Lambda(0))^{l_{1}+l_{2}+\ldots+l_{n}}}{l_{1} ! l_{2} ! \cdots l_{n} !} p_{01}^{l_{1}} \cdots p_{0 n}^{l_{n}} z_{1}^{l_{1}} \cdots z_{n}^{l_{n}} \prod_{j=1}^{n} \psi_{1 j}^{l_{1}} \cdots \psi_{n j}^{l_{n}}, \\
a_{2}(z, t)=\exp \left\{\sum_{i=1}^{n}\left(M_{i}(t)-M_{i}(0)\right) \frac{\alpha_{i}}{z_{i}}\right\}=\prod_{i=1}^{n} \exp \left\{\left(M_{i}(t)-M_{i}(0)\right) \frac{\alpha_{i}}{z_{i}}\right\}= \\
=\prod_{i=1}^{n} \sum_{q_{i}=0}^{\infty} \frac{\left[\left(M_{i}(t)-M_{i}(0)\right) \alpha_{i} z_{i}^{-1}\right]^{q_{i}}}{q_{i} !}=\sum_{q_{1}=0}^{\infty} \ldots \sum_{q_{n}=0}^{\infty} \prod_{i=1}^{n}\left[\left(M_{i}(t)-M_{i}(0)\right) \alpha_{i} z_{i}^{-1}\right]_{i}^{q_{i}} \\
q_{i} ! \\
=\sum_{q_{1}=0}^{\infty} \ldots \sum_{q_{n}=0}^{\infty}\left(M_{1}(t)-M_{1}(0)\right)^{q_{1}} \cdots\left(M_{n}(t)-M_{n}(0)\right)^{q_{n}} \frac{\alpha_{1}^{q_{1}} \cdot \ldots \cdot \alpha_{n}^{q_{n}}}{q_{1} ! \ldots \cdot q_{n} !} z_{1}^{-q_{1}} \cdots z_{n}^{-q_{n}},
\end{gathered}
$$




$$
\begin{gathered}
a_{3}(z, t)=\exp \left\{\sum_{i, j=1}^{n}\left(M_{i}(t)-M_{i}(0)\right) \beta_{i j} \frac{z_{j}}{z_{i}}\right\}=\prod_{i=1}^{n} \prod_{j=1}^{n} \exp \left\{\left(M_{i}(t)-M_{i}(0)\right) \beta_{i j} \frac{z_{j}}{z_{i}}\right\}= \\
=\prod_{i=1}^{n} \prod_{j=1}^{n} \sum_{r_{i}=0}^{\infty} \frac{\left[\left(M_{i}(t)-M_{i}(0)\right) \beta_{i j} z_{j} z_{i}^{-1}\right]^{i}}{r_{i} !}=\sum_{r_{1}=0}^{\infty} \cdot . \sum_{r_{n}=0}^{\infty} \prod_{i=1}^{n} \prod_{j=1}^{n} \frac{\left[\left(M_{i}(t)-M_{i}(0)\right) \beta_{i j} z_{j} z_{i}^{-1}\right]_{i}}{r_{i} !}= \\
=\sum_{r_{1}=0}^{\infty} \ldots \sum_{r_{n}=0}^{\infty}\left(M_{1}(t)-M_{1}(0)\right)^{r_{1}} \ldots\left(M_{n}(t)-M_{n}(0)\right)^{r_{n}} \frac{\left(\prod_{j=1}^{n} \beta_{1 j}\right)^{r_{1}} \cdot \ldots \cdot\left(\prod_{j=1}^{n} \beta_{n j}\right)^{r_{n}}}{r_{1} ! \cdots r_{n} !} \times \\
\times z_{1}^{r_{1}+r_{2}+\ldots+r_{n}} z_{2}^{r_{1}+r_{2}+\ldots+r_{n}} \cdots z_{n}^{r_{1}+r_{2}+\ldots+r_{n}} z_{1}^{-r_{1}} z_{2}^{-r_{2}} \cdots z_{n}^{-r_{n}}= \\
=\sum_{r_{1}=0}^{\infty} \ldots \sum_{r_{n}=0}^{\infty}\left(M_{1}(t)-M_{1}(0)\right)^{r_{1}} \ldots\left(M_{n}(t)-M_{n}(0)\right)^{r_{n}} \frac{\left(\prod_{j=1}^{n} \beta_{1 j}\right)^{r_{1}} \cdot \ldots \cdot\left(\prod_{j=1}^{n} \beta_{n j}\right)^{r_{n}}}{r_{1} ! \cdots r_{n} !} \times \\
\times z_{1}^{R-r_{1}} \cdots z_{n}^{R-r_{n}} .
\end{gathered}
$$

Multiplying $a_{0}(t), a_{1}(z, t), a_{2}(z, t), a_{3}(z, t)$ and $\prod_{l=1}^{n} z_{l}^{x_{l}}$ we obtain an expression (14).

\section{Example}

1. Suppose that the intensity $\lambda(t)=\lambda, \mu_{i}(t)=\mu_{i}, i=\overline{1, n}$ does not depend on time. In this case we have

$$
\begin{gathered}
\Lambda(t)=\lambda t, \Lambda(0)=0, M_{i}(t)=\mu_{i} t, M_{i}(0)=0, i=\overline{1, n}, \\
a_{0}(t)=\exp \left\{-\sum_{i=1}^{n}\left[\lambda p_{0 i}\left(1-\varphi_{i}\right)+\mu_{i}\left(1-\beta_{i i}\right)\right] t\right\} .
\end{gathered}
$$

Equation (14) takes the form

$$
\begin{aligned}
& \Psi_{n}(z, t)=a_{0}(t) \sum_{l_{1}=0}^{\infty} \ldots \sum_{l_{n}=0}^{\infty} \sum_{q_{1}=0}^{\infty} \ldots \sum_{q_{n}=0}^{\infty} \sum_{r_{1}=0}^{\infty} \ldots \sum_{r_{n}=0}^{\infty} \lambda^{\sum_{i=1}^{n} l_{i}} t^{\sum_{i=1}^{n}\left(l_{i}+q_{i}+r_{i}\right)} \times \\
& \times \prod_{i=1}^{n}\left[\frac{p_{0 i}^{l_{i}} \alpha_{i}^{q_{i}} \mu_{i}^{q_{i}+r_{i}}\left(\prod_{j=1}^{n} \psi_{i j}\right)^{l_{i}}\left(\prod_{j=1}^{n} \beta_{i j}\right)^{r_{i}}}{l_{i} ! q_{i} ! r_{i} !} z_{i}^{x_{i}+l_{i}-q_{i}-r_{i}+R}\right] .
\end{aligned}
$$


2. Let $\lambda(t)=\lambda t, \quad \mu_{i}(t)=\mu_{i}\left[\cos \left(\omega_{i} t\right)+1\right], \quad i=\overline{1, n}$. In this case $\Lambda(t)=\frac{\lambda}{2} t^{2}$,

$$
\begin{aligned}
\Lambda(0)=0, M_{i}(t)=\mu_{i}\left[\frac{\sin \left(\omega_{i} t\right)}{\omega_{i}}+t\right], M_{i}(0)=0, i=\overline{1, n}, \\
a_{0}(t)=\exp \left\{-\sum_{i=1}^{n}\left[\frac{\lambda p_{0 i}\left(1-\varphi_{i}\right)}{2} t^{2}+\mu_{i}\left(1-\beta_{i i}\right) t+\frac{\mu_{i}\left(1-\beta_{i i}\right)}{\omega_{i}} \sin \left(\omega_{i} t\right)\right]\right\}, \\
\times \prod_{i=1}^{n}\left[\frac{\left.\left.\Psi_{n i}(z, t)=a_{0}(t) \sum_{l_{i}=0}^{\infty} \ldots \sum_{l_{n}=0}^{\infty} \sum_{q_{1}=0}^{\infty} \ldots \sum_{q_{n}=0}^{\infty} \sum_{r_{i}=0}^{\infty} \ldots \sum_{r_{n}=0}^{\infty}\left(\frac{\lambda}{2}\right)^{\sum_{i=1}^{n} l_{i}} \mu_{i}^{x_{i}+l_{i}+\sum_{j=1}^{n} r_{j}-1} t^{\sum_{i=1}^{n} l_{i}} \times \prod_{j=1}^{n} \psi_{i j}\right)^{l_{i}}\left(\prod_{j=1}^{n} \beta_{i j}\right)^{r_{i}} \times\left(\frac{\sin \left(\omega_{i} t\right)}{\omega_{i}}+t\right)^{q_{i}+r_{i}} z_{i}^{x_{i}+l_{i}-q_{i}-r_{i}+R}\right] .}{l_{i} ! q_{i} ! r_{i} !} .\right.
\end{aligned}
$$

Let us assume, for example, that we have found, the probability of state $P(1,1, \ldots, 1, t)$. It is the coefficient of $z_{1} z_{2} \cdot \ldots \cdot z_{n}$ in the expansion of $\Psi_{n}(z, t)$ in multiple series (15). So the degree of $z_{i}$ must satisfy the relation $x_{i}+l_{i}-q_{i}-r_{i}+R=1, i=\overline{1, n}$. It follows that

$$
\begin{gathered}
q_{i}=x_{i}+l_{i}+\sum_{\substack{j=1 \\
j \neq i}} r_{j}-1, i=\overline{1, n}, \\
q_{i}+r_{i}=x_{i}+l_{i}+\sum_{j=1} r_{j}-1, i=\overline{1, n}, \\
l_{i}+q_{i}+r_{i}=x_{i}+2 l_{i}+\sum_{j=1} r_{j}-1, i=\overline{1, n}, \\
\sum_{i=1}^{n}\left(l_{i}+q_{i}+r\right)=\sum_{i=1}^{n}\left(x_{i}+2 l_{i}\right)+n(R-1) .
\end{gathered}
$$

Therefore, from (15) and (16) we get that in case 1): 


$$
\begin{aligned}
& P(1,1, \ldots, 1, t)=a_{0}(t) \sum_{l_{1}=0}^{\infty} \ldots \sum_{l_{n}=0}^{\infty} \sum_{r_{1}=0}^{\infty} \ldots \sum_{r_{n}=0}^{\infty} \lambda^{\sum_{i=1}^{n} l_{i}} t^{\sum_{i=1}^{n}\left(x_{i}+2 l_{i}\right)+n(R-1)} \times \\
& \times \prod_{i=1}^{n}\left[\frac{p_{0 i}^{l_{i}} \alpha_{i}{ }^{x_{i}+l_{i}+\sum_{\substack{j=1 \\
j \neq i}}^{n} r_{j}-1} \mu_{i}^{x_{i}+l_{i}+R-1}\left(\prod_{j=1}^{n} \psi_{i j}\right)^{l_{i}}\left(\prod_{j=1}^{n} \beta_{i j}\right)^{r_{i}}}{l_{i} ! r_{i} !\left(x_{i}+l_{i}+\sum_{\substack{j=1 \\
j \neq i}}^{n} r_{j}-1\right) !},\right.
\end{aligned}
$$

and in case 2 :

$$
\begin{aligned}
& P(1,1, \ldots, 1, t)=a_{0}(t) \sum_{l_{1}=0}^{\infty} \ldots \sum_{l_{n}=0}^{\infty} \sum_{r_{1}=0}^{\infty} \ldots \sum_{r_{n}=0}^{\infty}\left(\frac{\lambda}{2}\right)^{\sum_{i=1}^{n} l_{i}} t^{2 \sum_{i=1}^{n} l_{i}} \times
\end{aligned}
$$

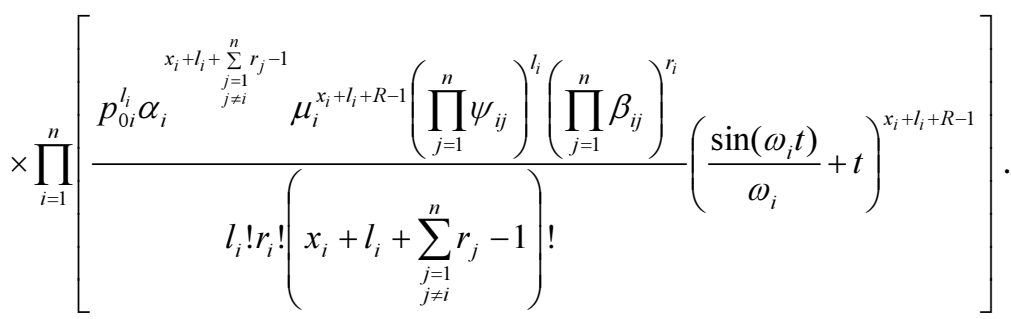

Suppose that $n=10$ and at the initial moment the network is in state $(2,2,2,2,2,2,2,2,2,2,0)$. Let us find the probability of the state $P(1,1,1,1,1,1,1,1,1,1, t)$ using the formula (15). Let $\lambda=20$, the transition probabilities of messages are equals: $p_{0 i}=0.1, i=\overline{1,10}, p_{10 i}=0.1, i=\overline{0,9}, p_{i 0}=0.5, i=\overline{1,9}, p_{i 10}=0.5, i=\overline{1,9}$, $p_{i i}=0, i=\overline{0,10}$. In addition, the intensity of the service of messages in the systems are equals: $\mu_{i}=20.8, i=\overline{1,4}, \mu_{i}=10.22, i=\overline{4,9} \quad \mu_{10}=20.5$. The conditional probabilities $\varphi_{i}, \psi_{i j}, \alpha_{i}, \beta_{i j}, i, j=\overline{1, n}$ are calculated according to (1)-(3), by the formulas

$$
\begin{gathered}
\phi_{i}=\left(1-f^{(i)}\right)\left(p_{i 0}+\sum_{j=1}^{n} p_{i j} \phi_{j}\right), \psi_{i j}=f^{(i)} \delta_{i j}+\left(1-f^{(i)}\right) \sum_{l=1}^{n} p_{i l} \psi_{l j}, \\
\alpha_{i}=p_{i 0}+\sum_{j=1}^{n} p_{i j} \phi_{j}, \beta_{i j}=\sum_{l=1}^{n} p_{i l} \psi_{l j}, i, j=\overline{1, n}
\end{gathered}
$$

is taken into account that $\alpha_{i}=1-\sum_{j=1}^{n} \beta_{i j}, \varphi_{i}+\sum_{j=1}^{n} \psi_{i j}=1, i, j=\overline{1, n}$. 
The expressions for the time-dependent state probabilities was obtained on the computer using the mathematical calculation package software Wolfram Mathematica. Figure 1 shows a chart of this probability depending on the $t$ called

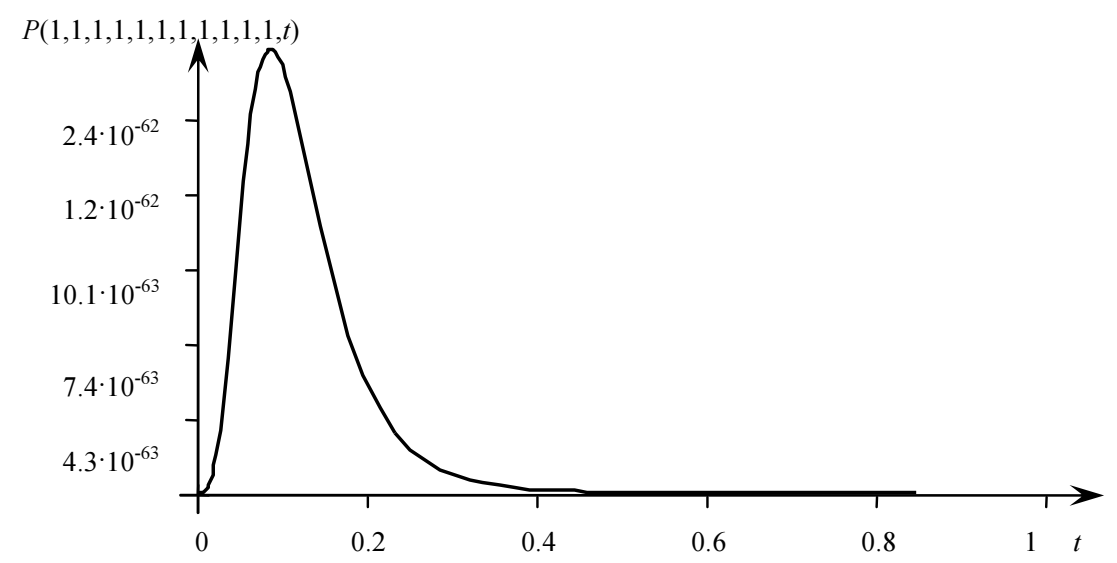

Fig. 1. The chart of the probability of the state $P(1,1,1,1,1,1,1,1,1,1, t)$

\section{References}

[1] Ivnitskii V., Theory of Queuing Networks, Fizmatlit, M. 2004, (in Russian).

[2] Malinkovskii Y., Queueing networks with bypass of nodes, Automation and Remote Control 1991, 2, 102-110 (in Russian).

[3] Malinkovskii Y., Yakubovich O., Queuing networks with applications serviced immediately. A models with a single type of applications, Automation and Remote Control 1998, 1, 98-106 (in Russian).

[4] Matalytski M., Queuing theory and its applications, M. Matalytski, O. Tikhonenko, E. Koluzaeva, Grodno 2011 (in Russian). 